УдК

\title{
АНАЛИЗ ЭФФЕКТИВНОСТИ МАССОВОЙ МІМО СИСТЕМЫ ВОСХОДЯЩЕЙ СВЯЗИ ПРИ ИСПОЛЬЗОВАНИИ КАНАЛА С ЗАМИРАНИЯМИ НАКАГАМИ-М
}

\author{
М. АЛЬ-РАВИ ${ }^{1}$, М. АЛЬ-РАВИ ${ }^{2}$ \\ ${ }^{1}$ Университет Ибб, Йемен, Ибб, Ибб сити \\ ${ }^{2}$ Университет Аль-Мустансирии, Ирак, Багдад, Урбан
}

\begin{abstract}
Аннотация. В статье исследована эффективность рабочих характеристик массовой MIMO (massive MIMO) системы восходящей связи (uplink) при использовании канала с замираниями Накагами-m. Эффективность измерена как зависимость спектральной эффективности от количества антенн базовой станции при различных значениях параметра $m$. Использованы две схемы линейного детектирования: объединение с максимальным отношением MRC (maximum ratio combining) и приемник с обращением в нуль незначащих коэффициентов ZF (zero forcing). Результаты моделирования показали, что при возрастании параметра $m$ спектральная эффективность увеличивается медленно, а при увеличении числа антенн базовой станции однако она увеличивается существенно. Кроме того, спектральная эффективность при использовании схемы ZF оказывается лучше, чем в случае использования схемы MRC.
\end{abstract}

Ключевые слова: массовая МIMO; канал с замираниями Накагами- $m$

\section{1. ВВЕДЕНИЕ}

В системах беспроводной связи использование многоэлементных антенн как на стороне передатчика, так и на стороне приемника, дает возможность существенно повысить надежность канала связи и увеличить общую пропускную способность системы с помощью технологии МІМО. Эта технология использована в различных стандартах беспроводной связи, таких как беспроводные системы третьего (3G), четвертого ( $4 \mathrm{G})$ и пятого $(5 \mathrm{G})$ поколений. Хотя большинство современных МIMO систем используют 2-8 антенн, предложенные в последнее время массовые MIMO системы (massive MIMO) [1], нацелены на использование потенциально большого увеличения пропускной способности, которое появится при применении более крупных массивов антенн.

«Массовое MIMO» (massive MIMO), известное также как «большая антенная систе- ма», «очень большое MIMO» (very large MIMO), «гипер MIMO» (hyper MIMO) и «полноразмерное MIMO» (full-dimension MIMO), представляет собой новую развивающуюся технологию, которая увеличивает МIMO на порядок по сравнению с существующей современной технологией. Технология «массовое MIMO» представляет собой очень большую многопользовательскую МІМО систему, в которой используется много антенных решеток, которые одновременно обслуживают большое число терминалов одиночных антенн (в общем случае системы могут быть оснащены многоэлементными антеннами) при одним и том же время-частотном ресурсе.

Базовой предпосылкой применения технологии «массовое МIMO» является использование всех достоинств традиционной технологии MIMO, но в значительно большем масштабе. В целом, массовое МIMO делает возможным 


\section{БИБЛИОГРАФИЧЕСКИЙ СПИСОК}

1. Marzetta T. L. Noncooperative cellular wireless with unlimited number of base station antennas / Thomas L. Marzetta // IEEE Trans. Wireless Commun. - Nov. 2010. - Vol. 9, No. 11. - P. 3590-3600. - DOI : 10.1109/TWC.2010.092810.091092.

2. Ngo H. Q. Performance bounds for very large multiuser MIMO systems / Hien Quoc Ngo // M.Sc. Thesis, Linköping University. - Sweden, 2012.

3. Measured propagation characteristics for very-large MIMO at $2.6 \mathrm{GHz} / \mathrm{Xiang}$ Gao, Fredrik Tufvesson, Ove Edfors, Fredrik Rusek // Signals, Systems, and Computers : 46th Annual Conf. ASILOMAR, 4-7 Nov. 2012, USA : proc. — IEEE, 2012. - DOI : 10.1109/ACSSC.2012.6489010.

4. Ashikhmin A. Pilot contamination precoding in multi-cell large scale antenna systems / Alexei Ashikhmin, Thomas L. Marzetta // Information Theory : IEEE Int Symp. ISIT, 1-6 Jul. 2012, Cambridge : proc. - IEEE, 2012. - DOI : 10.1109/ISIT.2012.6283031.

5. Pakdeejit E. Linear precoding performance of massive MU-MIMO downlink system / Eakkamol Pakdeejit// M.Sc. Thesis, Linköping University. — Sweden, 2013. - URL : http://www.diva-portal.org/smash/get/ diva2:630516/FULLTEXT01.pdf.
6. Massive MIMO for next generation wireless systems / Erik G. Larsson, Ove Edfors, Fredrik Tufvesson, Thomas L. Marzetta // IEEE Commun. Magazine. - Feb. 2014. - Vol. 52, No. 2. - P. 186-195. - DOI : 10.1109/MCOM.2014.6736761.

7. Scaling up MIMO: opportunities and challenges with very large arrays / Fredrik Rusek, Daniel Persson, Buon Kiong Lau, Erik G. Larsson, Thomas L. Marzetta, Ove Edfors, Fredrik Tufvesson // IEEE SP Magazine (Signal Processing). - Jan. 2013. - Vol. 30, No. 1. - P. 40-60. - DOI : 10.1109/MSP.2011.2178495.

8. Ngo H. Q. Energy and spectral efficiency of very large multiuser MIMO systems / Hien Quoc Ngo, Erik G. Larsson, Thomas L. Marzetta // IEEE Trans. Commun. Apr. 2013. — Vol. 61, No. 4. - P. 1436-1449. — DOI : 10.1109/TCOMM.2013.020413.110848.

9. Choi J. Downlink training techniques for FDD massive MIMO systems: Open-loop and closed-loop training with memory / Junil Choi, David J. Love, Patrick Bidigare // IEEE J. Selected Topics Signal Processing. Oct. 2014. - Vol. 8, No. 5. - P. 802-814. - DOI : 10.1109/JSTSP.2014.2313020.

10. Ali M. Massive MIMO for fifth generation wireless system / M. Ali, et al. // B. Sc. Project, University of Ibb. - Yemen, 2014

11. Ngo H. Q. Massive MIMO: fundamental and system design / H. Q. Ngo // Dissertation, Linköping University. - Sweden, 2015. — URL : http://liu.se/elliit/ artiklar-3/1.681525/MassiveMIMO_FundamentalsandSy stemDesigns.pdf.

Поступила в редакцию ?

\title{
PERFORMANCE OF MASSIVE MIMO UPLINK SYSTEM OVER NAKAGAMI-M FADING CHANNEL
}

\author{
${ }^{1}$ Ibb University, Ibb, Yemen \\ ${ }^{2}$ Al-Mustansiriya University, Baghdad, Iraq \\ *e-mail: muhrawi@yahoo.com \\ **e-mail: muaayed@yahoo.com
}

This paper studies the performance of massive MIMO uplink system over Nakagami- $m$ fading channel. The performance is measured in terms of spectral efficiency versus the number of base station antennas with different values of the parameter $m$ using two schemes of linear detection; maximum ratio combining (MRC) and zero forcing receiver (ZF). The simulation results show that as $m$ increases, the spectral efficiency increases slowly, but it increases significantly with the rise of the number of base station antennas. It should be also noted that the spectral efficiency with ZF is better than that with MRC.

Keywords: massive MIMO; Nakagami- $m$ fading channel 